First publ. in: Physica / B [Condensed Matter], Vols. 249-251 (1998), pp. 660-663

\title{
Contact phenomena in 2D electron systems
}

\author{
V. Shikin ${ }^{a \cdot *}$, E. Teskeb, P. Leiderer ${ }^{c}$, P. Wyder ${ }^{h}$

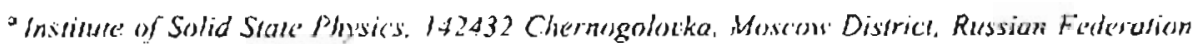

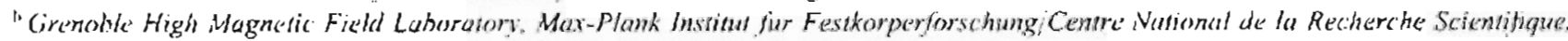 \\ B.P.160, F.38042, Grenohle, Ceder 9. France

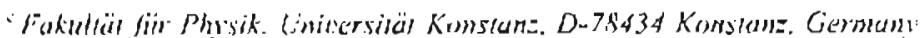

\begin{abstract}
A promising way to investigale 2D contact phenomena is proposed. This method is based on the idea of depositing surface state electrons (SSE) on a thin layer of liquid helium covering the surlace of a solid sample containing a 2D-charge carrier system. The density of SSE adjusts 10 screen contact-induced pesturbations of the electrostatic potential across the sample. As a result, the helium layer thickness varies due to the variation of the clectrostatic pressure, thus providing a map. This map may be read of interferometrically by a lechniquc already employed for the investigation of multi-electron dimples on helium. We have realized this mapping for a structured electrode as a lest sample 1o demonstrate the resolution of the method. i) 1998 Elsevier Science B.V. All rights reserved.
\end{abstract}

Keyords: Liquid helinm; Contact phenomena

Contact phenomena are well known in 3D metál anu semiconductor physics [1,2]. We have in mind for example the determination of the work function for different 3D metals, the solution of the Schottky barrier problem and the applications of this solution to different aspects of transistor physics, in particular the creation of heterostructures, etc. In all these cases the perturbation of the electron density near the boundary between the contacting metals or semiconductors is well localized within the so-called Debye radius.

\footnotetext{
* Corresponding author. Fax: + $3(1965764111$; e-mail: shikin $(a)$ issp.at.ru
}

The same reasons as in $3 D$ systems lead to electron density contacl perturbations in 2D conducting structures. However. due to the peculiarities of low-dimensional screening this perturbation falls off as $1: x$ and hence has no special localisation length. As a result, the use of metallic source-drain terminals, which is typical of $2 \mathrm{D}$ transport measurements, leads to the perturbations of the electron density practically along the entire 2D system. It is evident that this phenomenon is very important for different transport problems in low-dimensional electron systems, for example the Quantum Hall Effect, different size effects, eic. 
One promising way for the investigation of $2 \mathrm{D}$ contact phenomena is based on the idea of deposiling electrons on a liquid helium film condensed onco the surface of a $2 \mathrm{D}$ sample in the presence of metallic terminals. The density of this classical 2DEG which adjusts to screen the potential from the sample provides a map. This charge density map may be read by optically measuring the variation in film thickness produced by the electrostatic pressure due to the charges, a technique alseady employed for the investigation of multi-electron dimples on helium [3].

One favourable detail of the presented method is its realisation under DC conditions. The existing alternative technique based on the linear clectrooptic effect already used for the mapping of $2 \mathrm{D}$ potential distributions [4-6] needs an $A C$ perlurbation. Besides, the electrons on a helium film could realise the mapping without total screening of the 2D electric potential distribution while the linear electro-optic effect is realised in the presence of an additional gate only.

In this paper, we investigate the possible use of charged helium films for the mapping of model potential perturbations and demonstrate that this technique proves to be very promising for the applications outlined above.

1. Let us start from the solution of the electrostatic problem for a gated Corbino sample schematically shown in Fig. 1.

In the presence of clectrompotential difference $\Delta V$ between the stripe "1" and terminals " 2 ". "3" wc have the following electron density distributions along the Corbino system for difierent screening levels (sec Fig. 2a-c). If Corbino is practically ungated (Fig. 2a), the extra electrons are distributed mainly between the electrodes $2 \cdots 1-3$. The integral neutrality requirement is then fulfilled without taking the gate into account.

In the opposite limit (Fig. 2c) the gate screening comes in, and the central Corbino parl " $\mathrm{"} \mathrm{has} \mathrm{an}$ essential, practically uniform fraction of cxtra electrons. The corresponding compensation charge is distributed mainly along the gate. This interesting peculiarity of $2 \mathrm{D}$ contact phenomena is importanl for the interpretation of many experimental results, e.g., the edge nature of minimal magnetocapacitance [7], unusual SdH oscillations in a

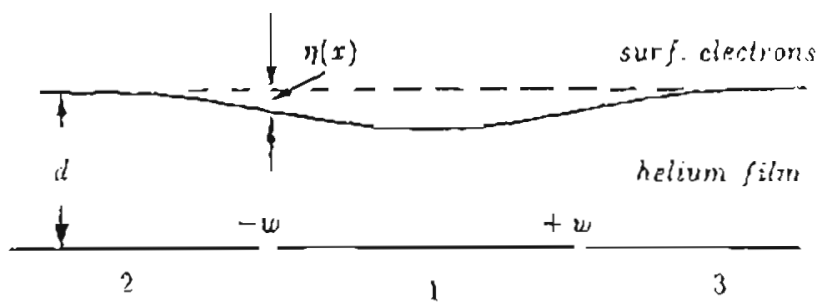

Fig. 1. Geometry and notations of the mapping problems with the electrons on helium film.

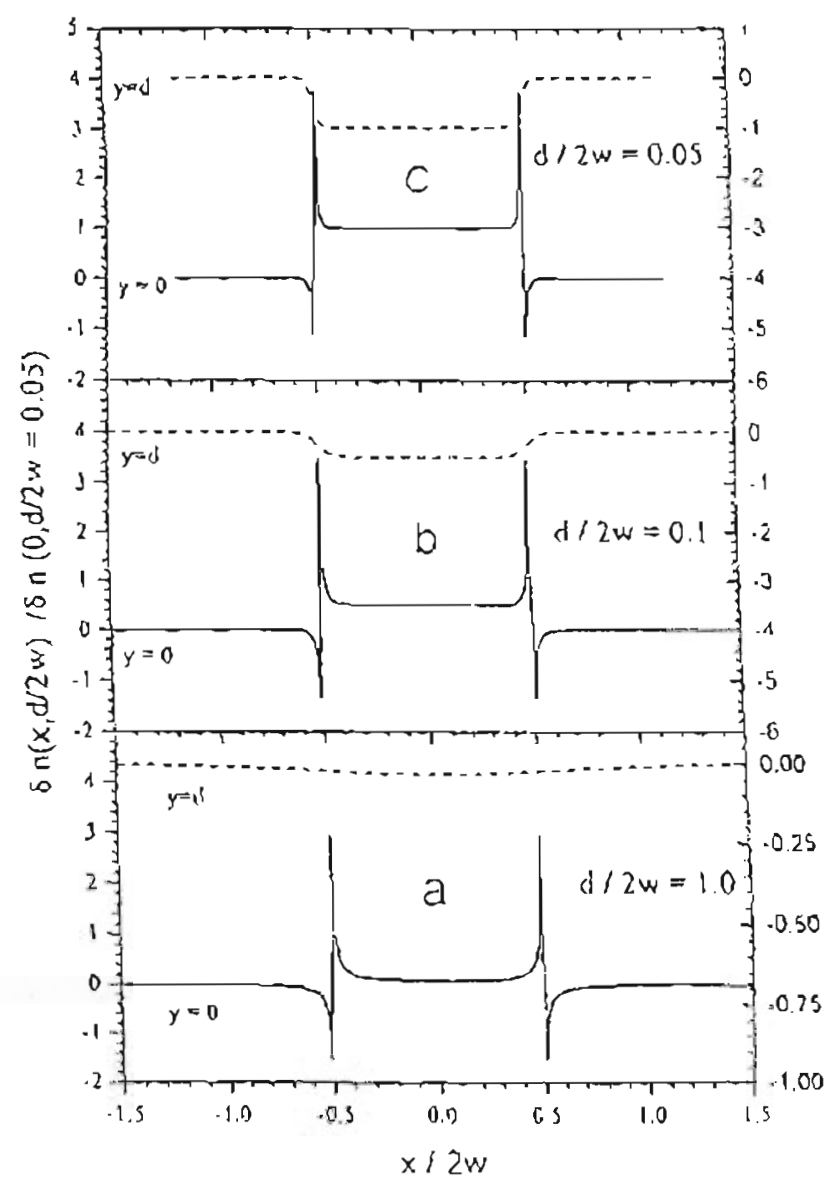

Fig. 2. Charge distribution along the gated Corbino disk with the potential perturbation between the electrodes 2-1-3 for

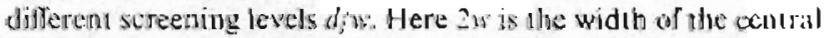
Corbine part, $d$ is the thickness of the helum film dolelectric spacer). Solid lines correspond to the Corbino sample. Dashed lines show the gate charge distribution. All density distributions are normalised to the distribution with the ratio $d i w=0.01$.

gated Corbino disk [8], etc. The same density distribution peculiarity is important for the potential mapping by electrons on a helium film. 

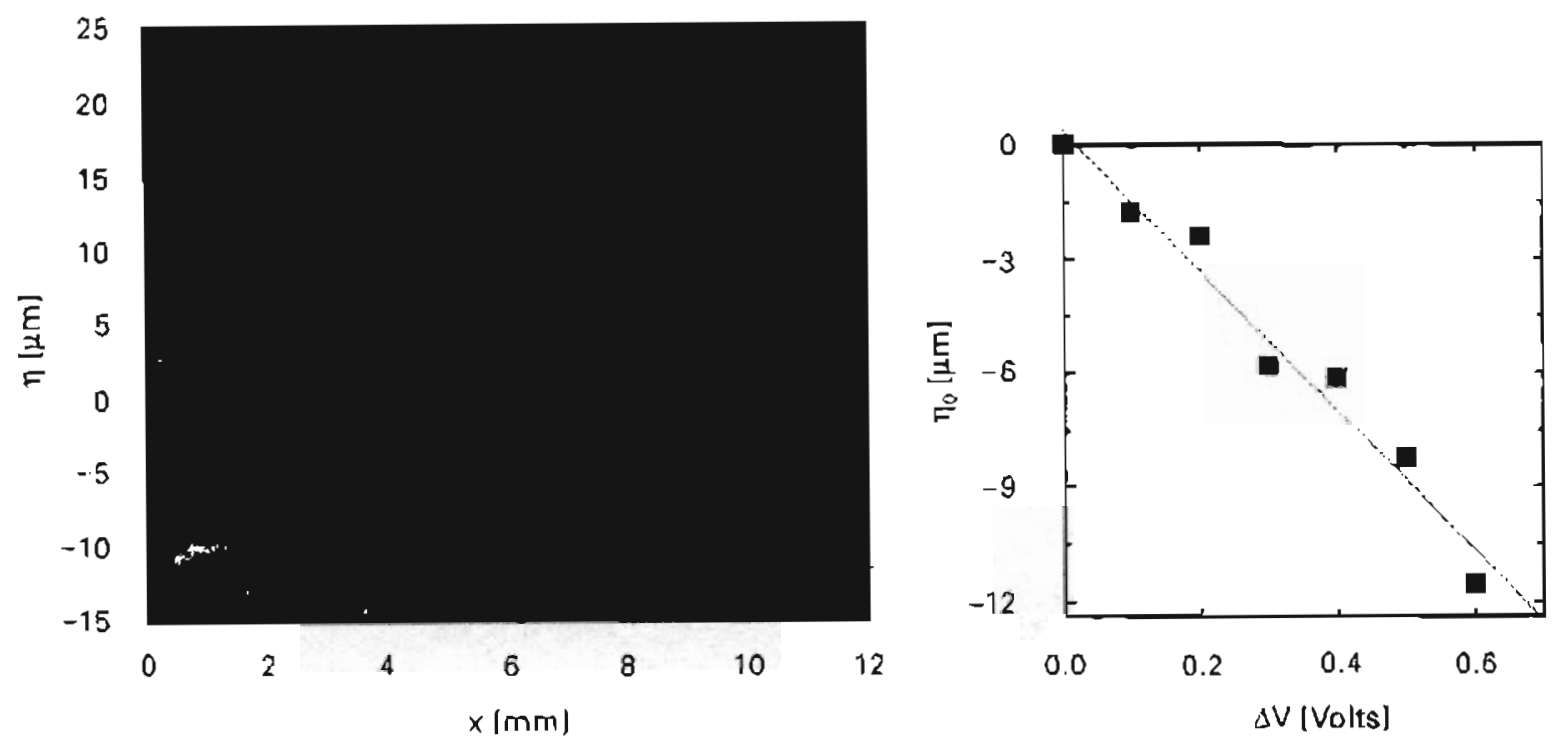

Fig. 3. Left: Calculations compared to the experimental image. Four perturbations in these figures correspond to different $x:(1,0.5,0.2$ and $0.1 \mathrm{~mm}$ ). Right: Measured helium film deformation depth against the applied perturbation voltage $\Delta V$ for $2 \mathrm{w}=1 \mathrm{mim}$. $d=100 \mu \mathrm{m}$.

2. The point is that a non-uniform electron density distribution along the gate leads to a nonuniform deformation of the helium film, which could cover the Corbino sample (see Fig. 1) and this deformation can be detected optically using well-known methods $[3 ; 9]$. Therefore, the mapping of non-uniform potential perturbations along the 2D electron system by electrons on the helium film is reduced to two steps. First, a charged helium film with a given 2D system as a substrate is prepared, and second the optical technique [3,9] is applied to study the helium film deformation caused by a non-uniform electron distribution along the helium film due to the screening redistribution of these electrons in the presence of potential perturbations in the $2 \mathrm{D}$ electron system. Realisation of this program for the system shown in Fig. 1 is presented in Fig. 3 where the left picture shows the omparison between the optical image of the deformed helium film via $\Delta V \neq 0$ perturbation and corresponding calculations using the solution of Poisson and mechanic equilibrium equations. The right panel in Fig. 3 demonstrates a linear behavjour of the helium film deformation versus applied voltage $\Delta V$. The solid line in this figure corresponds to the calculations without adjustable parameters.
3. The above information leads to the conclusion that mapping of $2 \mathrm{D}$ contact perturbations by electrons on a liquid helium film has good prospects. This method is suitable for a general presentation of the potential map. Besides, it can be useful to extract quantitative information about the details of low-dimensional contact phenomena.

Some qualitative conclusions follow from the curves plotted in Fig. 2. These results show that in the absence of an additional gate a non-uniform electron density is developed along the entire 2D electron system. In the presence of an additional gate, this perturbation is mainly uniform, but it still extends along the whole 2D system.

This activity is partly supported by INTAS 93939 and by NASA-PSA NAS 15-10110, project TM-17.

\section{References}

[1] L.D. Landau, E.M. Lifshiz, Ejectrodynamics of Continous Media Moscow. 1957.

[2] S.M. Sze. Physics of Semiconductor Devices, Wiloy New York, 1981.

[3] P. Leiderer. W. Ebner. V. Shikin. Surf. Sci. 11.3 (1982] 405. 
[4] P.F. Fontein, P. Hendriks, F.A.P. Blom, I.K. Wolter: L.I. Giling, C.W.I. Beenaker, Surf. Sci. 963 (1992) 91.

[S] R. Knott, W. Dietsche, K.v. Klitzing, K. Plong, K. Eberle. Semicond. Sci. Technol. 10 (1995) 117.

[6] W. Dietsche K.v. Klitzing, U. Ploog. Suri. Sci. 361/362 (1996) 289
[7] S. Takaoka, K. Oto, H. Kurimoto, K. Murase, K. Gama, S. Nishi, Phys. Rev. Lcti. 72 (1994) 3080.

[8] V.T. Dolgopolov, A.A. Shashkin. G.V. Kraychenko et al., Pis'ma Zh. EKsp. Teor. Fiz. 63 (1996) 55.

[9] D. Savignac, P. Leiderer, Phys. Rey. Lett. 49 (1982) 1869. 